\title{
Caracterización de los estilos de aprendizaje de estudiantes de Ingeniería Industrial de la UCEVA - Colombia
}

\section{Characterization of the learning styles of Industrial Engineering students at UCEVA}

BOLAÑOS VALENCIA, William de $\mathrm{J}^{1}$

SANDOVAL, Edgar de J. ${ }^{2}$

LASSO CARDONA, Luis A. ${ }^{3}$

\begin{abstract}
Resumen
Esta investigación caracterizo los Estilos de Aprendizaje (EA) de estudiantes y docentes del programa de Ingeniería Industrial de la UCEVA. Se aplicó el cuestionario CHAEA a una muestra de 130 estudiantes. Se identificó que el EA predominante es el reflexivo. Se verifico con el coeficiente de Pearson y la prueba $t$ de student que la edad y el género no son significativos en el EA preferido. Por otra parte, el EA preferido por los docentes es el teórico.

Palabras clave: aprendizaje, estilos de aprendizaje, CHAEA
\end{abstract}

\begin{abstract}
This research characterized the Learning Styles (EA) of students and teachers of the UCEVA Industrial Engineering program. The CHAEA questionnaire was applied to a sample of 130 students. The predominant $A E$ was identified as reflexive. It was verified with the Pearson's coefficient and the student's t test that age and gender are not significant in the preferred EA. On the other hand, the EA preferred by teachers is the theoretical.
\end{abstract}

key words: learning, learning styles, CHAEA

\section{Introducción}

La dinámica empresarial de hoy, altamente competitiva demanda profesionales con capacidades acordes que les permita enfrentarse a nuevos retos y contribuir a consolidar los sistemas productivos. Por ello, es necesario evaluar la formación académica recibida por los estudiantes desde las primeras etapas, garantizando que los procesos de enseñanza-aprendizaje sean de alta calidad y adaptados a la forma en que ellos mismos llevan a cabo su aprendizaje, de tal forma que se corrija la pobre apropiación y capacidad de explicación de los fenómenos y poderlos entender de manera sistemática (González-Cardona y Morales-Pinzón, 2020).

Recientes estudios evidenciaron que el modelo educativo tradicional dejo de ser acertado, y que no respondía a los nuevos requerimientos de la sociedad. En particular se observó que los titulados carecían de capacidades

\footnotetext{
${ }^{1}$ Ingeniero Industrial, Universidad Tecnológica de Pereira, Colombia. Magister en Administración, Universidad del Valle, Colombia. Profesor Facultad de Ingeniería Unidad Central del Valle del Cauca, Colombia. wbolanos@uceva.edu.co

${ }^{2}$ Ingeniero de Sistemas, Universidad Antonio Nariño, Colombia. M. Sc. in Virtual Education and ICT, Universitat Oberta de Catalunya. Profesor Facultad de Ingeniería Unidad Central del Valle del Cauca, Colombia. esandoval@uceva.edu.co

${ }^{3}$ Ingeniero de Sistemas, Universidad del Valle, Colombia. M. Sc. Gestión de la Tecnología Educativa, Universidad de Santander, Colombia. Profesor Asistente Universidad del Valle, Colombia. luis.lasso@correounivalle.edu.co
} 
para desenvolverse en el campo laboral, sumado esto a la poca relación entre la academia y el sector productivo. Razón por la cual, la Organización para la Cooperación y el Desarrollo Económico (OCDE) exhortó un cambio de paradigma educativo acorde a un modelo constructivista, que permita al estudiante empoderarse de su conocimiento y aplicarlo a la vida real (Aragón y Jiménez, 2009).

Tradicionalmente los procesos de enseñanza-aprendizaje han estado basados en la conceptualización de diversas corrientes del pensamiento. En espacial en las ciencias de la educación la teoría conductista, cognitivista y constructivista, y las teorías emergentes de Gagné, Bandura y Luria, han labrado el camino de la educación hasta principios del siglo XXI (Huamaní, 2018).

En este sentido, el enfoque acostumbrado de enseñanza que pone al maestro como el eje central del conocimiento es cosa del pasado, y modelos cognitivistas y constructivistas que relacionen el pensum con problemas reales $y$, donde se construya aprendizaje de forma colaborativa son recibidos con mayor motivación (Alzahrani, 2017).

Identificar la manera en que los estudiantes obtienen conocimiento es fundamental, ya que permite la formulación de nuevos contenidos curriculares que favorezcan una formación acorde a las necesidades de la sociedad actual y la actualización de estrategias pedagógicas por parte de los maestros (Coto, 2020), puesto que sea logrado evidenciar que una educación de calidad está estrechamente relacionada con el nivel de preparación de los docentes (Padilla y Mayoral, 2020).

Para ayudar a identificar las tendencias referentes a como se capacitan los estudiantes, varios autores han diseñado instrumentos que incluyen diversos factores relacionados con el proceso de aprendizaje. Uno de éstos, es el Cuestionario Honey-Alonso Estilos de Aprendizaje (CHAEA) empleado en todos los niveles educativos (Gutiérrez-Tapias, 2018).

Esta investigación propone la caracterización de los Estilos de Aprendizaje de los estudiantes del programa de Ingeniería Industrial de la Unidad Central del Valle del Cauca (UCEVA, Colombia), en todo su ciclo de formación aplicando el CHAEA, para lo cual se requiere inicialmente aclarar dos conceptos básicos que fundamentan el estudio: Aprendizaje y Estilos de Aprendizaje (EA). Cabe resaltar que la definición de EA debe fundamentarse en las teorías del aprendizaje, y que tales teorías se refieren a aquellos constructos que tratan de explicar cómo se aprende (Briceño, 2016).

\subsection{Marco referencial}

Aunque no existe una definición de Aprendizaje aceptada por todos los teóricos e investigadores, Alonso, Gallego, y Honey (1997) lo definen básicamente como un cambio relativamente permanente de la conducta producto de la práctica o experiencia de una actividad que repercute en el capacidad del hombre e influye en su crecimiento y madurez.

Igualmente, la definición del concepto EA también es bastante estudiada. Shunk (2012), básicamente lo define como el tratamiento que llevan a cabo los aprendices para descubrir, organizar, tratar y consultar la información. Castro y Guzmán (2005), agregan que los EA están enmarcados en diferencias cualitativas y cuantitativas individuales resultado de factores cognitivos y motivacionales que afectan la manera de aprender del individuo, y Velasco-Yañez (1996), menciona que características biológicas, sociales, motivacionales y ambientales son factores fundamentales que también definen su potencial cognitivo. Por otra parte, Arias-Gallegos (2011) y Hernández, Rodríguez y Vargas (2012) recalcan que fundamentar la educación en EA implica primero identificar tales estilos en los estudiantes, para después diseñar contenidos ajustados de aprendizaje. 
En la década de los 70's David Kolb y varios de sus colaboradores desarrollaron lo que ellos llamarón la teoría del aprendizaje cíclico y que más tarde se conoció como el Modelo de Kolb, en el cual el Aprendizaje es concebido como una espiral continua de cuatro etapas, y que también permite clasificar los EA según el comportamiento del aprendiz (Fuentes-Olavarría, 2019). Estas etapas son: 1) Activo: aprende haciendo y trabaja en grupo, 2) Reflexivo: son observadores, analizan, y ven diversas perspectivas del problema, 3) Teórico: prefieren las teorías antes de actuar, son independientes y analíticos y, 4) Pragmático: son prácticos y prefieren la aplicación de lo aprendido a un problema real (Rodríguez-Cepeda, 2018).

Por otra parte, es significativo resaltar la importancia del uso de estrategias metodológicas -entendidas como un conjunto de actividades que por su estructura orgánica incrementa la probabilidad de obtener un determinado logro (Montenegro, 2003), dentro del proceso pedagógico para que respondan a los diferentes EA, de manera que se favorezca el aprendizaje de los estudiantes de acuerdo con su ciclo evolutivo, nivel de desarrollo y necesidades educativas, con la finalidad de elevar los niveles de motivación, fortalecer la calidad de los aprendizajes y conseguir un mejor rendimiento académico (Díaz-Mosquera, 2012).

\subsection{Estado del arte}

Como estrategias encaminadas a disminuir las tasas de deserción, alcanzar las competencias requeridas en los estudiantes y lograr mayor motivación en su desempeño académico, algunas universidades han desarrollado investigaciones y encontrado una correlación significativa entre el rendimiento académico y el EA. Tal es el caso en la Universidad Tecnológica de Bolívar donde se analizó los EA de estudiantes de segundo semestre (Ruiz, Trillos y Morales, 2006), en una Universidad chilena con estudiantes de primer año de enfermería (González, Sáez y Ramírez, 2016), en 2009 en la Universidad de León, España se identificó que el estilo predominante era el reflexivo y teórico (López y Silva, 2009), y en el instituto superior pedagógico privado "Nuestra Señora de Guadalupe" de la provincia de Huancayo-Perú (De Mola, 2008).

De igual forma, se han realizado varios estudios que permiten caracterizar los EA en estudiantes de Ingeniería Industrial. Por ejemplo, en la Universidad Técnica del Norte en Ecuador, se encontró que el estilo predominante era el pragmático (Cala, Riera-García y Jaramillo, 2014). En Universidad Militar Nueva Granada se analizó a estudiantes de segundo semestre encontrándose que no hay un EA predominante (Lagos, Berdugo y Cogollo, 2019), y en la Universidad Autónoma de Occidente el estudio reflejo que el EA dominante fue el reflexivo-teórico (Ortiz-Soto, y Medina-Figueroa, 2017).

\section{Metodología}

Para el desarrollo de la investigación se realizó un diseño descriptivo-comparativo de diferencias entre grupos. Se tomó como población los estudiantes del programa de Ingeniería Industrial de la UCEVA cuyo rango de edades oscilan entre 15 y 25 años, y docentes responsables de las asignaturas que forman parte de los semestres 1, 2, 5 y 9 . Además, se definieron dos metodologías de trabajo: caso 1 y caso 2.

En el caso 1, se identificaron los EA de los estudiantes de 1 a 9 semestre. Se utilizó un muestreo aleatorio simple en el cual el error de medición fue del $5 \%$, nivel de confianza del $90 \%$ y la proporción de individuos que poseen la característica objeto de estudio en la población de referencia fue $50 \%$. Por tanto, el tamaño de muestra $\mathrm{n}=$ 130 encuestas.

En el caso 2, se caracterizaron los EA de estudiantes de semestres de dos ciclos de formación: área de ciencias básicas ( 1 y 2 semestre), y el área de formación profesional (5 y 9 semestre).

Con los estudiantes de primer semestre se buscó identificar el estado inicial de aprendizaje teniendo en cuenta su proceso de formación y madurez académica en la etapa del colegio; el segundo semestre para evaluar la 
transición de la formación secundaria a la universitaria; el quinto semestre, para evaluar su madurez académica al finalizar los estudios de ciencias básicas; el noveno semestre, para evaluar su desarrollo profesional en el transcurso de la carrera.

Además, en el proceso de investigación se planteó la caracterización de los EA de los docentes de las asignaturas definidas en el caso 2. De igual manera, el análisis de los syllabus de las asignaturas de los semestres 1, 2, 5 y 9 , objeto de estudio, que permitiera identificar información sobre las metodologías didácticas utilizadas.

El objetivo de esta información será contrastar los EA de los estudiantes, los docentes y las estrategias didácticas utilizadas para observar algún tipo de correspondencia y plantear acciones de mejora o articulación en el proceso de enseñanza aprendizaje.

\subsection{Cuestionario de evaluación}

La caracterización de los EA de estudiantes y docentes se realizó utilizando el CHAEA, compuesto por 80 preguntas, de las cuales 20 corresponden a cada EA. Además, el cuestionario fue complementado con información de variables sociodemográficas como edad, sexo, carácter de colegio de egreso (en el caso de estudiantes). Adicionalmente, años de experiencia laboral y carácter de la universidad de egreso. Estos resultados se contrastaron con el baremo general propuesto por Alonso, Domingo, Gallego y Honey, a fin de identificar el EA predominante en la población.

\section{Resultados}

Los resultados obtenidos en esta investigación se organizan en tres módulos discriminados como Información de estudiantes, Información de docentes y Metodologías didácticas. De igual manera, para cada subgrupo de estos se desarrolla un análisis estadístico de las variables que permitan hacer una inferencia y proponer acciones que permitan alinear los EA de los docentes y estudiantes.

\subsection{Información de estudiantes}

Caso 1 (tamaño de muestra $n=147$ ). El grafico 1 muestra que el estilo de mayor preferencia de los estudiantes es el reflexivo.

Gráfico 1

EA predomínate de los estudiantes

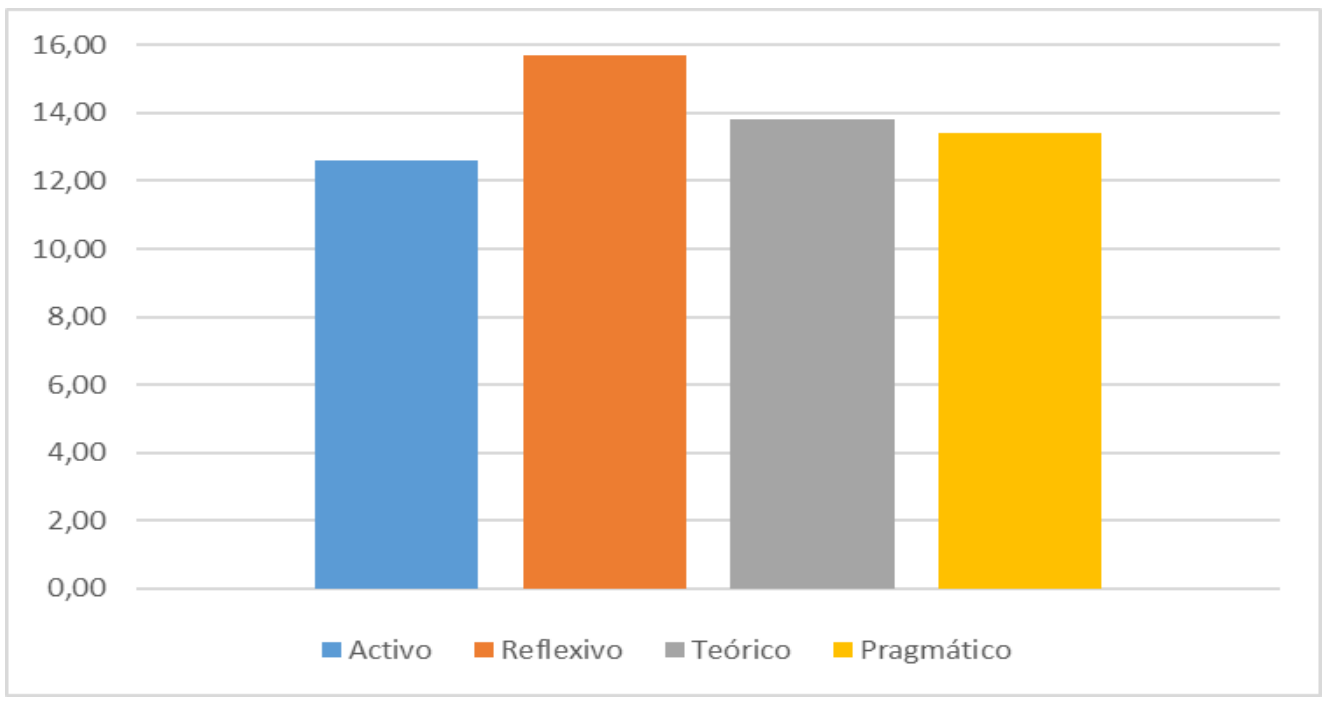

Fuente: autores 
Caso 2 (caracterización por semestres). La tabla 1 evidencia que el EA predominante es el reflexivo con una ponderación moderada. Además, a pesar de ser una población diferente, no se identifica un cambio en su EA, partiendo del supuesto que en la medida que avanzan en su proceso de formación profesional hay variables que podrían influir como, por ejemplo: diferentes metodologías pedagógicas utilizadas por los docentes, la edad de los estudiantes y el grado de madurez profesional.

Tabla 1

EA de los estudiantes (semestres 1, 2, 5 y 9)

\begin{tabular}{|c|c|c|c|c|c|c|c|c|c|c|}
\hline \multirow{3}{*}{ EA } & \multicolumn{8}{|c|}{ Semestre } & \multirow{3}{*}{$\begin{array}{c}\text { P. } \\
\text { Promedio }\end{array}$} & \multirow{3}{*}{ Preferencia } \\
\hline & \multicolumn{2}{|c|}{1} & \multicolumn{2}{|c|}{2} & \multicolumn{2}{|c|}{5} & \multicolumn{2}{|c|}{9} & & \\
\hline & $P$ & $\mathrm{~V}$ & $P$ & $\mathrm{~V}$ & $P$ & $\mathrm{~V}$ & $P$ & V & & \\
\hline Activo & 12 & $M$ & 13 & $A$ & 12 & $M$ & 12 & $M$ & 12,1 & M \\
\hline Reflexivo & 16 & $M$ & 15 & $M$ & 13 & B & 15 & $M$ & 14,5 & M \\
\hline Teórico & 14 & $A$ & 14 & $A$ & 12 & $M$ & 13 & $M$ & 13,3 & M \\
\hline Pragmático & 14 & $A$ & 14 & $A$ & 13 & $M$ & 13 & $M$ & 13,3 & M \\
\hline
\end{tabular}

$\mathrm{P}=$ Puntación. $\mathrm{V}=$ Valoración. $\mathrm{M}=$ Moderada. $\mathrm{B}=$ Baja. $\mathrm{A}=$ Alta

Fuente: autores

\section{Análisis estadístico de los EA por semestre}

Para el caso 2 se realizó un análisis comparativo entre semestres que permitiera evaluar la significancia en la preferencia de los EA de los estudiantes en la medida que avanzan los semestres de formación académica. En especial, evaluar si en el área de ciencias básicas y el área de formación profesional se mantiene el EA, y si entre estas dos grandes áreas se evidencia un cambio significativo derivado posiblemente por variables como la edad y el género.

Para hallar las diferencias en la puntuación media de los EA en función del semestre cursado por el estudiante, se desarrolló un análisis estadístico univariable comparativo con una prueba $\mathrm{F}$ de Fisher y un nivel de significación de 0,05 . En este proceso se compararon los valores del estadístico de prueba y el valor crítico registrado. Para ello, se compararon los semestres 1 y 2 ; luego, los semestres 5 y 9 ; posteriormente, los cuatros semestres entre sí. Esto se hizo para cada uno de los cuatro EA.

Además, se planteó la hipótesis para cada caso para determinar la existencia de diferencias significativas en la puntuación media del EA de cada estudiante.

Entonces:

- Prueba de hipótesis para el semestre 1 y 2 :

Hipótesis nula: entre los semestres 1 y 2 no existen diferencias significativas con respecto a la puntuación media.

Hipótesis alternativa: entre los semestres 1 y 2 si existen diferencias significativas con respecto a la puntuación media.

Decisión:

F de prueba < F crítico, se acepta la hipótesis nula.

$\mathrm{F}$ de prueba $\geq \mathrm{F}$ crítico, se rechaza la hipótesis nula. 
- Prueba de hipótesis para el semestre 5 y 9 :

Hipótesis nula: entre los semestres 5 y 9 no existen diferencias significativas con respecto a la puntuación media

Hipótesis alternativa: entre los semestres 5 y 9 si existen diferencias significativas con respecto a la puntuación media

Decisión:

$\mathrm{F}$ de prueba < F crítico, se acepta la hipótesis nula.

$\mathrm{F}$ de prueba $\geq \mathrm{F}$ crítico, se rechaza la hipótesis nula.

- Prueba de hipótesis para cuatro semestres: 1, 2, 5 y 9

Hipótesis nula: entre los semestres 1, 2, 5 y 9 no existen diferencias significativas con respecto a la puntuación media

Hipótesis alternativa: entre los semestres 1, 2, 5 y 9 si existen diferencias significativas con respecto a la puntuación media

Decisión:

F de prueba < F crítico, se acepta la hipótesis nula.

$\mathrm{F}$ de prueba $\geq \mathrm{F}$ crítico, se rechaza la hipótesis nula.

La tabla 2 resume los cálculos obtenidos para el estadístico de prueba y el valor critico obtenido de la tabla F, con las características respectivas. Teniendo en cuenta estos criterios y los resultados obtenidos se concluye lo siguiente:

- Comparativo entre los semestres 1 y 2: los valores calculados para $\mathrm{F}$ son menores que los valores de $\mathrm{F}$ crítico, por tanto, se acepta la hipótesis nula; o sea, la diferencia en la preferencia de los estilos de aprendizaje no es significativa

- Comparativo entre los semestres 5 y 9, los valores calculados para $\mathrm{F}$ son menores que los valores de $\mathrm{F}$ crítico, por tanto, se acepta la hipótesis nula; o sea, la diferencia en la preferencia de los estilos de aprendizaje no es significativa

Comparativo entre todos los cuatro semestres $(1,2,5$ y 9), los valores calculados para $\mathrm{F}$ son menores que los valores de F crítico, por tanto, se acepta la hipótesis nula; o sea, la diferencia en la preferencia de los estilos de aprendizaje no es significativa.

Tabla 2

Comparativo estadístico de prueba y el valor critico de $\mathrm{F}$ por EA de cada semestre

\begin{tabular}{|l|c|c|c|c|c|c|}
\hline \multirow{2}{*}{\multicolumn{1}{|c|}{ EA }} & \multicolumn{2}{|c|}{ Semestre 1 y } & \multicolumn{2}{c|}{ Semestre 5 y 9 } & \multicolumn{2}{c|}{ Semestres 1, 2, 5 y 9 } \\
\cline { 2 - 7 } & $\begin{array}{c}\text { Estadístico } \\
\text { prueba F }\end{array}$ & Valor & Estadístico & Valor \\
critico F & prueba F & critico F & Estadístico & Valor \\
prueba F & critico F \\
\hline Activo & 0,812 & 4,171 & 0,003 & 4,196 & 0,123 & 2,769 \\
\hline Reflexivo & 1,168 & 4,171 & 1,906 & 4,196 & 2,064 & 2,769 \\
\hline Teórico & 0,280 & 4,171 & 1,967 & 4,196 & 1,732 & 2,769 \\
\hline Pragmático & 0,830 & 4,171 & 0,580 & 4,196 & 0,378 & 2,769 \\
\hline
\end{tabular}

Fuente: autores

\section{EA por semestre y por género}

El gráfico 2 presentan los resultados que identifican los EA de los estudiantes por semestre y género. En general, se observa que tanto hombres y mujeres prefieren el EA reflexivo, con una leve preferencia por los estilos reflexivos y teóricos en los hombres de semestre 2. La preferencia por el EA reflexivo puede suceder por el efecto 
que se genera con el uso de estrategias didácticas por parte de los docentes en los diferentes semestres que van desarrollando habilidades que fortalecen las características de un EA de este tipo.

Gráfico 2

Consolidado EA de los estudiantes

por semestre y género

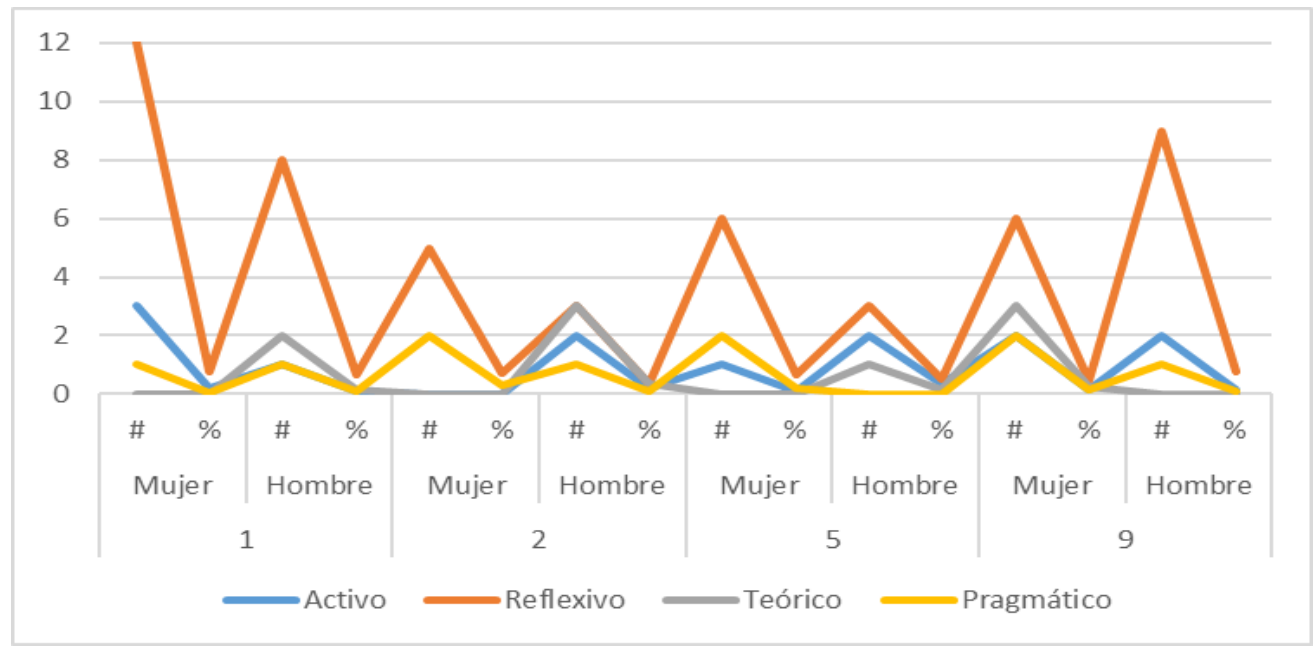

Fuente: autores

\section{Análisis estadístico para evaluar la relación entre el EA, la edad y el género}

A continuación, se presentan las pruebas estadísticas y análisis de correlación para determinar la significancia entre las variables edad, género y el EA de los estudiantes, para lo cual se aplicaron pruebas t-Student para variables independientes, un análisis de varianza bifactorial con un nivel de significancia del $5 \%$. En este análisis se tomaron los semestres como poblaciones independientes.

Pruebas de hipótesis: esta prueba se planteó para cada EA

- Hipótesis nula, Ho: no hay diferencias entre las medias de las preferencias en los EA según la edad.

- Hipótesis Alternativa, Ha: si hay diferencias entre las medias de las preferencias en los EA según la edad.

Criterios de decisión para significancia de edad y género:

- Si el estadístico $t \geq$ valor critico de $t$, entonces, rechazo la hipótesis nula.

- Si el estadístico $t<$ valor critico de $t$, entonces, no rechazo la hipótesis nula.

La tabla 3 presentan los resultados de los criterios de decisión para evaluar la significancia de la edad en el EA.

Tabla 3

Análisis de significancia de la edad de los estudiantes en el EA

\begin{tabular}{|l|c|c|c|c|c|c|c|c|}
\hline \multirow{2}{*}{ EA } & \multicolumn{2}{|c|}{ Semestre 1 } & \multicolumn{2}{c|}{ Semestre 2 } & \multicolumn{2}{c|}{ Semestre 5 } & \multicolumn{2}{c|}{ Semestre 9 } \\
\cline { 2 - 9 } & $\mathrm{t}$ & $\begin{array}{c}\text { Valor } \\
\text { critico t }\end{array}$ & $\mathrm{t}$ & $\begin{array}{c}\text { Valor } \\
\text { critico t }\end{array}$ & $\mathrm{t}$ & $\begin{array}{c}\text { Valor } \\
\text { critico t }\end{array}$ & $\begin{array}{c}\text { Valor } \\
\text { critico } \mathrm{t}\end{array}$ \\
\hline Activo & 9,7178 & 2,0049 & 6,9397 & 2,0423 & 7,4760 & 2,0452 & 15,4792 & 2,0106 \\
\hline Reflexivo & 3,6927 & 2,0049 & 5,4322 & 2,0423 & 5,7464 & 2,0452 & 10,5784 & 2,0106 \\
\hline Teórico & 6,4818 & 2,0049 & 7,1958 & 2,0423 & 8,9357 & 2,0452 & 13,7752 & 2,0106 \\
\hline Pragmático & 7,3138 & 2,0049 & 4,8894 & 2,0423 & 11,0469 & 2,0452 & 13,3873 & 2,0106 \\
\hline
\end{tabular}

Fuente: autores 
Se observa que para cada uno de los cuatro EA y los semestres objeto de estudio, el valor del estadístico de prueba es mayor que el valor critico de t; por tanto, se acepta la hipótesis alternativa.

\section{Significancia del género en el EA}

A continuación, se plantean pruebas de hipótesis para cada estilo de aprendizaje para determinar si hay o no diferencias significativas con respecto al género.

Prueba de hipótesis:

- Hipótesis nula, Ho: no hay diferencias entre las medias de las puntuaciones en los EA y el género.

- Hipótesis alternativa, Ha: si hay diferencias entre las medias de las puntuaciones en los EA y el género

Tabla 4 presentan los resultados de los criterios de decisión para evaluar la significancia del género en el EA.

Tabla 4

Análisis de significancia del género

de los estudiantes en el EA

\begin{tabular}{|l|c|c|c|c|c|c|c|c|}
\hline \multirow{2}{*}{ EA } & \multicolumn{2}{|c|}{ Semestre 1 } & \multicolumn{2}{c|}{ Semestre 2 } & \multicolumn{2}{c|}{ Semestre 5 } & \multicolumn{2}{c|}{ Semestre 9 } \\
\cline { 2 - 10 } & $\mathrm{t}$ & $\begin{array}{c}\text { Valor } \\
\text { critico } \mathrm{t}\end{array}$ & $\mathrm{t}$ & $\begin{array}{c}\text { Valor } \\
\text { critico } \mathrm{V}\end{array}$ & $\mathrm{t}$ & $\begin{array}{c}\text { Valor } \\
\text { critico t }\end{array}$ & $\begin{array}{c}\text { Valor } \\
\mathrm{critico} \mathrm{t}\end{array}$ \\
\hline Activo & 0,4786 & 2,0555 & $-0,7551$ & 2,1448 & $-0,3538$ & 2,1604 & $-0,4660$ & 2,0687 \\
\hline Reflexivo & $-0,9950$ & 2,0555 & 1,6979 & 2,1448 & $-0,1853$ & 2,1604 & $-1,4976$ & 2,0687 \\
\hline Teórico & $-1,8190$ & 2,0555 & 0,3252 & 2,1448 & $-1,5439$ & 2,1604 & 0,3906 & 2,0687 \\
\hline Pragmático & $-0,8786$ & 2,0555 & 0,3292 & 2,1448 & $-0,3681$ & 2,1604 & $-0,4447$ & 2,0687 \\
\hline
\end{tabular}

Fuente: autores

Se observa que para cada uno de los EA y los semestres objeto de estudio, el valor del estadístico de prueba es menor que el valor critico de t; por tanto, se acepta la hipótesis nula.

\section{Análisis de regresión y correlación}

También, en la investigación se aplicó la prueba de Pearson, entre la puntuación de los EA y la edad de los estudiantes permitiera estimar el nivel de relación entre estas variables. Con el cálculo de este coeficiente de correlación se buscó medir la fuerza de la relación lineal entre las variables X y Y en la muestra seleccionada. Para ello, se plantearon las siguientes hipótesis:

- Hipótesis nula, Ho: $r=0$; No existe una correlación lineal entre el EA y la edad $(r=0)$. El coeficiente de correlación obtenido procede de una población cuya correlación es cero.

- Hipótesis alternativa, Ha: $r \neq 0$. Si existe una correlación lineal entre el EA y la edad El coeficiente de correlación obtenido procede de una población cuyo coeficiente de correlación es distinto de cero.

Regla de decisión:

- $\mathrm{Si}|\mathrm{t}|>\mathrm{t}$ critico entonces rechace Ho. Concluye que hay una relación lineal.

- $\mathrm{Si}|\mathrm{t}| \leq \mathrm{t}$ critico entonces no rechace Ho. Concluye que no hay una relación lineal

El gráfico 3 presenta los resultados del cálculo del coeficiente de correlación aplicado para determinar la relación entre los EA y la edad de los estudiantes. 
Gráfico 3

Coeficiente de correlación entre

los EA y edad de los estudiantes

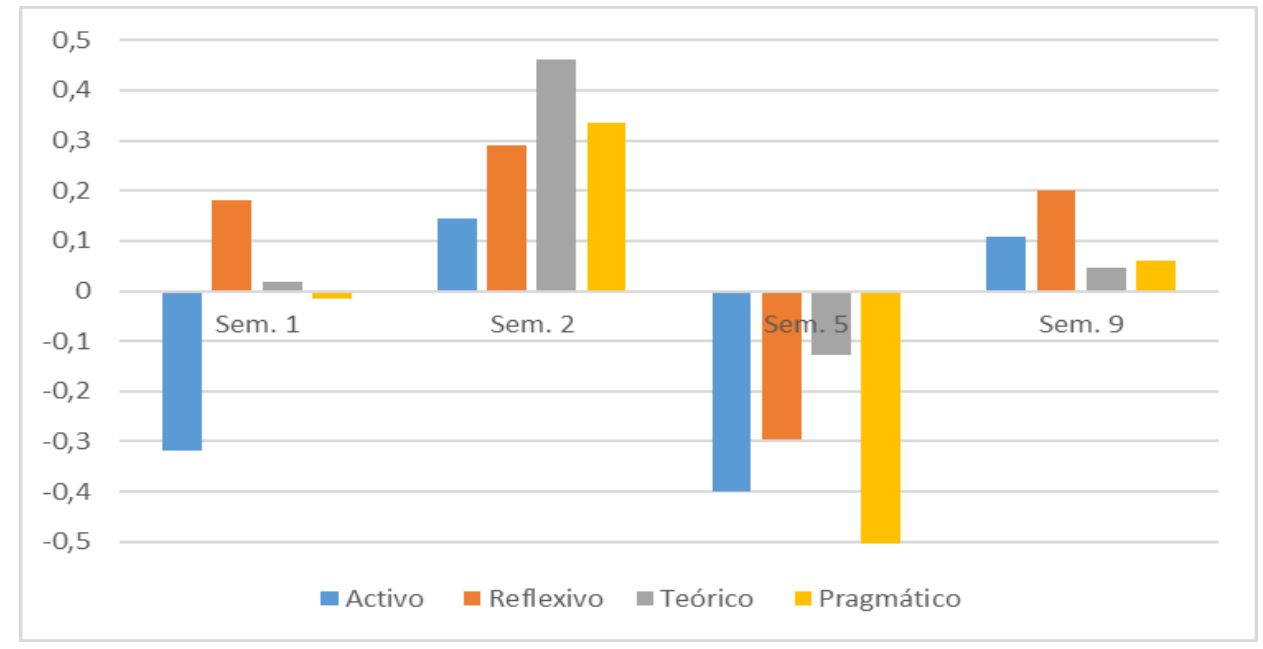

Fuente: autores

Tabla 5

Valores de estadístico $t$ y valor crítico para $r$

\begin{tabular}{|l|c|c|c|c|c|c|c|c|}
\hline \multirow{2}{*}{\multicolumn{1}{|c|}{ EA }} & \multicolumn{2}{|c|}{ Semestre 1 } & \multicolumn{2}{c|}{ Semestre 2 } & \multicolumn{2}{c|}{ Semestre 5 } & \multicolumn{2}{c|}{ Semestre 9 } \\
\cline { 2 - 9 } & $\mathrm{t}$ & $\begin{array}{c}\text { Valor } \\
\text { critico } \mathrm{t}\end{array}$ & $\mathrm{t}$ & $\begin{array}{c}\text { Valor } \\
\text { critico } \mathrm{t}\end{array}$ & $\mathrm{t}$ & $\begin{array}{c}\text { Valor } \\
\text { critico } \mathrm{t}\end{array}$ & $\mathrm{t}$ & $\begin{array}{c}\text { Valor } \\
\text { critico } \mathrm{t}\end{array}$ \\
\hline Activo & $-1,7055$ & 2,055 & 0,5452 & 2,1448 & 1,9448 & 2,1604 & 0,5166 & 2,0687 \\
\hline Reflexivo & 0,9400 & 2,0555 & 1,1381 & 2,1448 & $-1,1136$ & 2,1604 & 0,9861 & 2,0687 \\
\hline Teórico & 0,0938 & 2,0555 & 1,9448 & 2,1448 & $-0,4668$ & 2,1604 & 0,2175 & 2,0687 \\
\hline Pragmático & $-0,0734$ & 2,0555 & 1,3357 & 2,1448 & $-2,1146$ & 2,1604 & 0,2950 & 2,0687 \\
\hline
\end{tabular}

Fuente: autores

Teniendo en cuenta los resultados de la Figura 3 y en la Tabla 5, se observa que, en primer semestre hay una correlación lineal muy baja en los EA y la edad. Sin embargo, en el EA activo y pragmático se observa que hay una correlación negativa entre las variables.

En segundo semestre hay una correlación lineal baja entre las variables. Con respecto al primer semestre, hay una mejor correlación lineal. Sin embargo, se observa una correlación positiva en los cuatro EA y la edad.

En el quinto semestre se observa una correlación lineal moderada entre estas dos variables. Con respecto al segundo semestre, la relación es un poco más fuerte. Asimismo, existe una correlación negativa en los cuatro EA y la edad.

En noveno semestre hay una correlación lineal muy baja entre las dos variables. Comparando con el quinto semestre, desmejoró esta relación. Es muy parecida esta relación a la observada en el primer semestre. Se observa una correlación positiva en los cuatro EA y la edad.

En general se observa que para los cuatros semestres analizados, hay una relación lineal muy baja entre la puntuación de cada EA y la edad, es decir, la variable edad no guarda una relación lineal con el EA. Se puede 
afirmar, con cierta probabilidad, que el coeficiente de correlación procede de una población cuyo valor es cero. Igualmente, de acuerdo a los criterios de decisión $\mathrm{t} \geq \mathrm{t}$ crítico, no se rechaza Ho. Es decir, no existe significancia en la regresión lineal de las dos variables para la población analizada.

\subsection{Información de docentes}

El gráfico 4 presenta la caracterización de los EA de docentes asignados en las asignaturas de los semestres objeto de estudio. De acuerdo a estos resultados, se observa que el EA de mayor preferencia es el teórico con una valoración alta según el baremo general, pero con una leve excepción en segundo semestre donde EA preferido es el reflexivo. También se identifica que, en el ciclo de formación de ciencias básicas de ingeniería, los docentes caracterizados tienen EA diferentes (teórico y reflexivo), mientras que, en el ciclo de formación profesional su EA preferido es teórico, obtenido en el promedio general.

Gráfico 4

Estilos de aprendizaje de los docentes

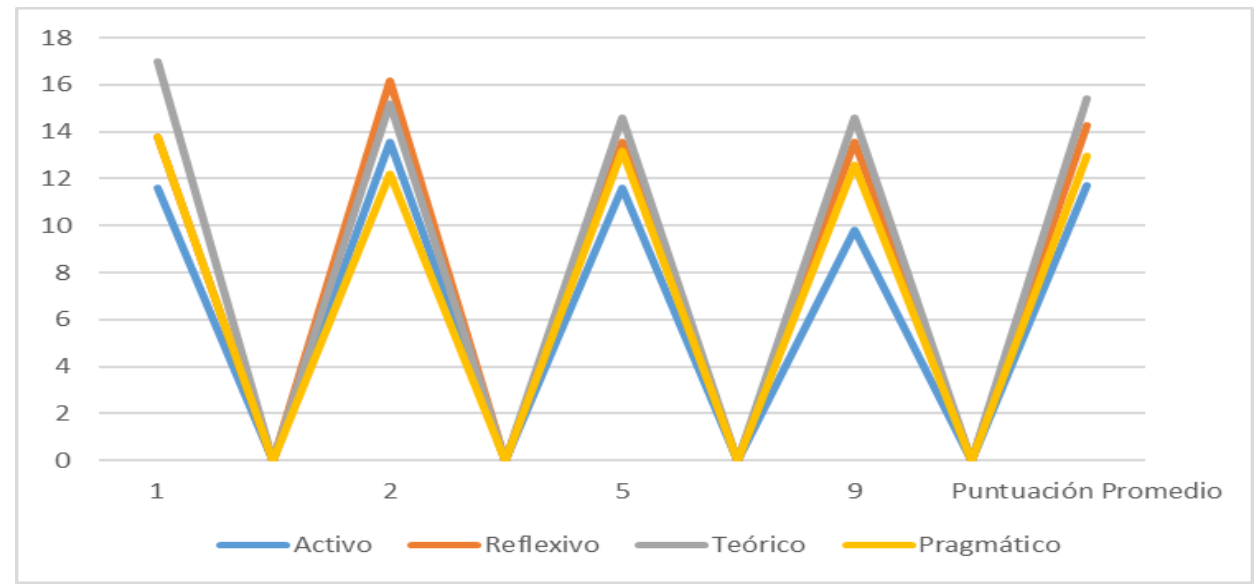

Fuente: autores

\section{Información de las estrategias metodológicas de enseñanza}

El gráfico 5 presenta las estrategias empleadas por los docentes según información de los syllabus de las asignaturas de los semestres objeto de estudio. Se aprecia entonces que la clase magistral es la estrategia más utilizada en todos los semestres. Le siguen las lecturas programadas y la consulta bibliográfica. También, se observa que las exposiciones y prácticas de laboratorio son las estrategias más utilizadas en los semestres de formación profesional; y los talleres y consultas bibliográficas en los semestres de formación básica.

Es importante tener en cuenta que las estrategias metodológicas caracterizadas, corresponden a estrategias cognitivas que fortalecen la comprensión y aplicación conceptual. Para un próximo estudio se recomienda recolectar esta información a partir de una encuesta que será aplicada a los docentes y estudiantes de tal manera que se pueda contrastar los resultados con lo planeado en el syllabus y la utilización real en el aula de clases.

Además, se debe revisar la utilización de las actividades lúdicas en algunos cursos del área profesional como estrategia metodológica de aprendizaje, buscando transferir los conocimientos en un contexto real que fortalezca la experimentación, haciendo énfasis en las características de un EA reflexivo para que los estudiantes alcancen los propósitos de aprendizaje propuestos. 


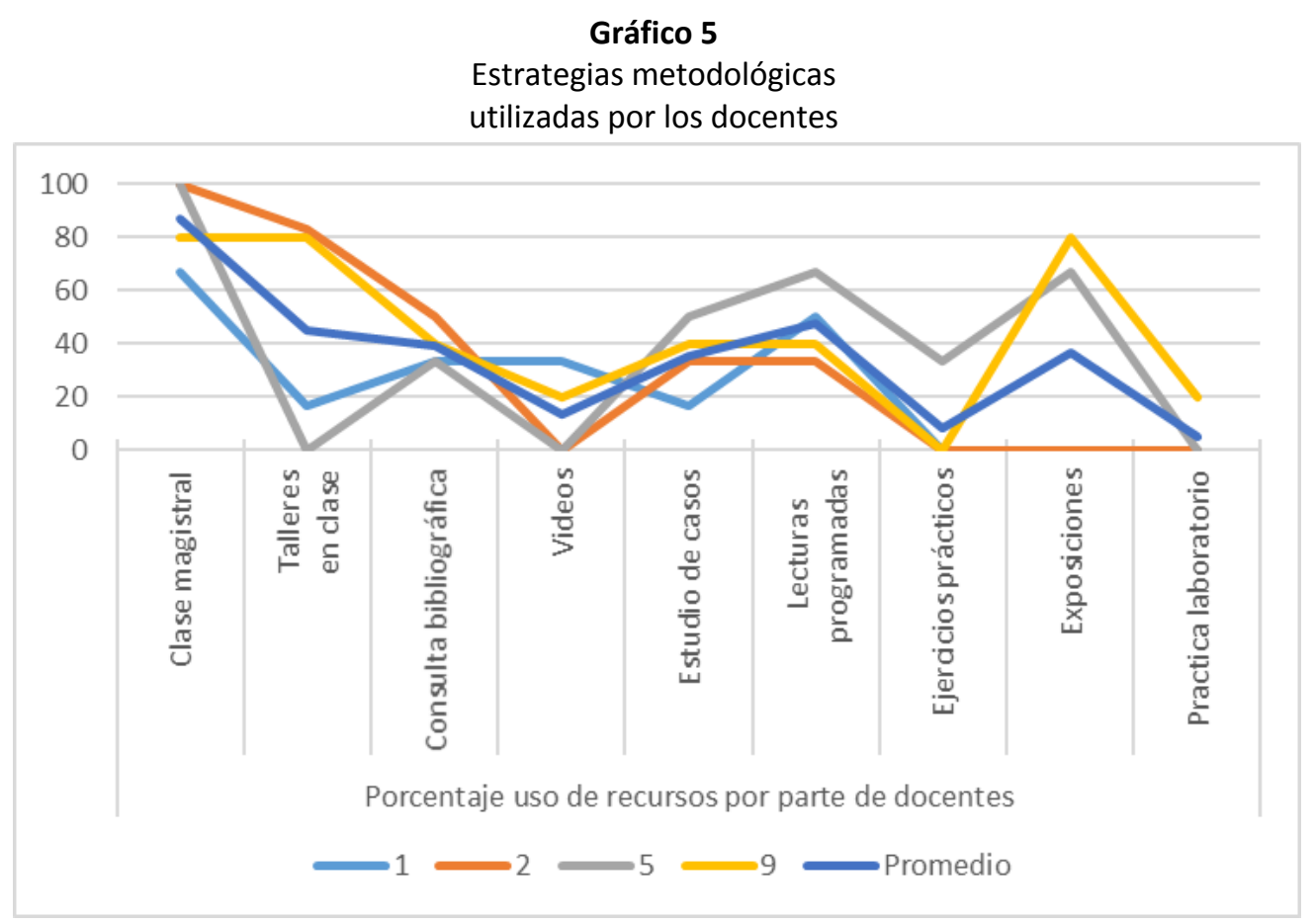

Fuente: Información recopilada de los SYLLABUS y organizada por los autores

\section{Conclusiones}

Los resultados obtenidos en el proyecto de investigación evidencian que el EA reflexivo es el preferido por los estudiantes. Esta tendencia permite identificar estudiantes receptivos, analíticos, que prefieran la aplicación de las ideas aprendidas en las aulas de clase, lo que implica una valiosa información para los docentes, que deban tener en cuenta esto al momento de diseñar las metodologías de enseñanza. De igual manera, la comparación entre los semestres del área básica de ingeniería y los semestres del área de formación profesional no se observaron diferencias significativas en la preferencia en los EA, ni tampoco, al comparar los cuatros semestres entre sí. Asimismo, no se encuentro evidencia contundente para afirmar que la edad y el género de los estudiantes esta correlacionada linealmente con la puntación media de los estilos de aprendizaje.

El contraste entre el EA de los estudiantes, donde predominó el reflexivo, y el de los docentes podría condicionar el desarrollo de las clases, en especial en el uso de estrategias metodológicas, situación que puede afectar el aprendizaje y su rendimiento académico. Por ello es fundamental capacitar a los docentes sobre los EA preferidos de los estudiantes y el impacto que estos tienen en los procesos de formación, para que sus estrategias pedagógicas se ajusten a la forma en que los estudiantes de hoy en día desean adquirir conocimientos que les ayuden a mejorar su perfil profesional (Hoffman, Ledesma y Liporace, 2017).

Es importante resaltar que cualquiera de los EA que se adopte, el proceso de enseñanza-aprendizaje tiene mejores resultados con la presencia del docente, cuyo papel se vuelve trascendental en el proceso de construcción de conocimientos por parte del alumno, y el alcance de ciertos objetivos formativos (García-Retana, 2013).

Finalmente, con los resultados de este trabajo se buscó contribuir al fortalecimiento de una de las líneas de investigación del grupo GEIPRO, que viene proponiendo metodologías lúdicas para ser empleadas en el proceso de enseñanza-aprendizaje haciendo la transferencia de conceptos en un contexto real con un entorno agradable y motivante para los dos actores del proceso. Igualmente, se espera que con un diseño adecuado de las 
actividades lúdicas se pueda responder eficazmente a los estudiantes de EA predominantes del programa de Ingeniería Industrial de la UCEVA.

\section{Referencias bibliográficas}

Alonso, C., Gallego, D. y Honey. P. (1995). Los estilos de aprendizaje. Procedimientos de diagnóstico y mejora. España: Ediciones Mensajero.

Alzahrani, M. (2017). The Effect of Using Online Discussion Forums on Students' learning. Turkish Online Journal of Educational Technology, 16(1), 164-176. Recuperado de: https://eric.ed.gov/?id=EJ1124930

Aragón, M. y Jiménez, Y. (2009). Diagnóstico de los estilos de aprendizaje en los estudiantes: Estrategia docente para elevar la calidad educativa. CPU-e, Revista de Investigación Educativa, 9. Recuperado de: http://www.uv.mx/cpue/num8/opinion/aragon_estilos_aprendizaje.html

Arias-Gallegos, W. (2011). Estilos de aprendizaje en estudiantes universitarios y sus particularidades en función de la carrera, el género y el ciclo de estudios. Rev. Estilos Aprendiz, 8(8), 112-135. Recuperado de: http://revistaestilosdeaprendizaje.com/article/view/939

Briceño, C. (2016). Estilos de aprendizaje de los estudiantes del Programa de Ingeniería Industrial y de Sistemas de la Universidad de Piura. Tesis de Maestría. Universidad de Piura, Perú. Recuperado de: https://hdl.handle.net/11042/2490

Cala, R., Riera-García, M. y Jaramillo, M. (2014). Determinación de los estilos de aprendizaje de estudiantes de 1er curso de Ingeniería Industrial y Electrónica de la Universidad Técnica del Norte. Ibarra, Ecuador. Revista Estilos de Aprendizaje, 7(14), 43-67. Recuperado de: http://revistaestilosdeaprendizaje.com/article/view/998/1706

Castro, S. y Guzmán, B. (2005). Los estilos de aprendizaje en la enseñanza y el aprendizaje: Una propuesta para su implementación. Rev. Invest., 58, 83-102. Recuperado de: https://www.redalyc.org/pdf/3761/376140372005.pdf

Coto, M. (2020). Descubrimiento del estilo de aprendizaje dominante en estudiantes de Matemática Superior. Revista Educación, 44(1). DOI: https://doi.org/10.15517/revedu.v44i1.38571

De Mola, J. (2008). Los estilos de aprendizaje de Honey-Alonso y el rendimiento académico en las áreas de formación general y formación profesional básica de los estudiantes del Instituto Superior Pedagógico Privado 'Nuestra Señora de Guadalupe' de la provincia de Huancayo. Revista Estilos de Aprendizaje, 1(1), 201-213. Recuperado de: http://revistaestilosdeaprendizaje.com/article/view/871

Díaz-Mosquera, E. (2012). Estilos de Aprendizaje. EIDOS, 5. DOI: https://doi.org/10.29019/eidos.v0i5.88

Hernández, C., Rodríguez, N. y Vargas, Á. (2012). Los hábitos de estudio y motivación para el aprendizaje de los alumnos en tres carreras de ingeniería en un tecnológico federal de la ciudad de México. Revista de la educación superior, 41(163), 67-87. Recuperado de:

http://www.scielo.org.mx/scielo.php?script=sci_arttext\&pid=S0185-27602012000300003\&lng=es\&tlng=es

Fuentes-Olavarría, D. (2019). Aportes del aprendizaje experiencial a la formación de estudiantes de enfermería en psiquiatría: Estudio cualitativo. Revista Mexicana de Investigación Educativa, 24(82), 833-851.

Recuperado de: http://www.scielo.org.mx/scielo.php?script=sci_arttext\&pid=S1405$66662019000300833 \& \operatorname{lng}=\mathrm{es} \&$ tlng=es 
García-Retana, J. (2013). Reflections on learning styles and calculus learning in engineering majors. Investig. en Educ., 13(1), 362-390. Recuperado de: http://www.scielo.sa.cr/scielo.php?script=sci_arttext\&pid=S1409$47032013000100014 \& \operatorname{lng}=$ en\&tlng=

González-Cardona, M. y Morales-Pinzón, T. (2020). Unidad didáctica y lúdica para explicar el fenómeno de contaminación del agua. Zona Próxima, 32, 75-104. Recuperado de: http://rcientificas.uninorte.edu.co/index.php/zona/article/download/12358/214421444371

González, F., Sáez, K. y Ramírez, J. (2016). Perfiles de estilos de aprendizaje y rendimiento académico en estudiantes de primer año de enfermería. Cienc. y Enferm., 22(1). Recuperado de: https://revistaschilenas.uchile.cl/handle/2250/52542

Gutiérrez-Tapias, M. (2018). Estilos de aprendizaje, estrategias para enseñar. Su relación con el desarrollo emocional y "aprender a aprender". Tendencias Pedagógicas, 31. Recuperado de: https://dialnet.unirioja.es/descarga/articulo/6383448.pdf

Hoffman, A., Ledesma, R. y Liporace, M. (2017). Estilos y estrategias de aprendizaje en estudiantes universitarios de Buenos Aires. Rev. Psicol., 35(2). 511-550. https://doi.org/10.18800/psico.201702.006

Huamaní, G. (2018). Estilos de aprendizaje de los estudiantes de la Facultad de Ingeniería Industrial y de Sistemas de la Universidad Nacional de Ingeniería. Tesis de Maestría. Universidad de Piura. Facultad de Ciencias de la Educación. Piura, Perú. Recuperado de: https://bit.ly/2yl04FG

Lagos, J., Berdugo, E. y Cogollo, J. (2019). Estilos de aprendizaje, un estudio en ingeniería a distancia. Rev. Vínculos, 16(2). Recuperado de: https://revistas.udistrital.edu.co/index.php/vinculos/article/download/15972/15497/

López, M. y Silva, E. (2009). Estilos de aprendizaje. Relación con motivación y estrategias. Revista Estilos de Aprendizaje, 4(4), 36-55. Recuperado de: https://www.ubu.es/sites/default/files/portal_page/files/documento_4_estilos_de_aprendizaje.pdf

Montenegro, I. (2003). Aprendizaje y Desarrollo de las Competencias, Editorial Magisterio, Bogotá.

Ortiz-Soto, L. y Medina-Figueroa, D. (2017). Caracterización del proceso de enseñanza-aprendizaje de la línea de administración de operaciones del programa de Ingeniería Industrial de la Universidad Autónoma de Occidente. Tesis. Universidad Autónoma de Occidente, Colombia. Recuperado de: https://bit.ly/3ffazLp

Padilla, I. y Mayoral, V. (2020). Las tutorías académicas en el fortalecimiento del álgebra en estudiantes de octavo grado en una escuela distrital de Barranquilla. Zona Próxima, 32, 33-54. Recuperado de: http://rcientificas.uninorte.edu.co/index.php/zona/article/view/11956/214421444369

Rodríguez-Cepeda, R. (2018). Los modelos de aprendizaje de Kolb, Honey y Mumford: implicaciones para la educación en ciencias. Sophia, 14(1), 51-64. DOI: https://doi.org/10.18634/sophiaj.14v.1i.698

Ruiz B., Trillos, J. y Morales, J. (2006) Estilos de aprendizaje y rendimiento academico en estudiantes universitarios. Revista galego-portuguesa de Psicoloxía e Educación, 13, 441-457. Recuperado de: https://ruc.udc.es/dspace/handle/2183/7034

Shunk, D. (2012) Teorías del aprendizaje: una perspectiva educativa. 6 Ed. México: Pearson Educación.

Velasco-Yáñez, S. (1996). Preferencias perceptuales de estilos de aprendizaje en cuatro escuelas primarias: Comparaciones y sugerencias para la formación y actualización de docentes. Revista Mexicana de Investigación Educativa, 1 (2). Recuperado de: http://redalyc.uaemex.mx/redalyc/pdf/140/14000203.pdf

Esta obra está bajo una Licencia Creative Commons

Attribución-NoCommercial 4.0 International 